\title{
OPTIMAL CONTROL OF FUNCTIONAL DIFFERENTIAL SYSTEMS*
}

\author{
F. COLONIUS $\dagger$ AND D. HINRICHSEN $\dagger$
}

\begin{abstract}
This paper presents a unified approach to diverse optimal control problems for hereditary differential systems (HDS). An abstract local maximum principle is established via the Dubovitskii-Milyutin method. It yields necessary conditions for the optimal control of HDS towards surfaces in $\boldsymbol{R}^{\boldsymbol{n}}$ and towards target sets in function spaces. Nondegeneracy criteria are included. It is shown that the necessary conditions are sufficient in the case of linear HDS with convex cost functionals. Analogous results are obtained for systems described by Fredholm equations with general control action. For Fredholm systems with targets in function spaces, the attainability space $\mathscr{A}$ is investigated, criteria for $\mathscr{A}$ to be closed are established and full attainability is characterized.
\end{abstract}

Introduction. This paper is mainly concerned with the optimal control of hereditary differential systems (HDS) with finite memory $h \geqq 0$ described by functional differential equations of the form:

$$
\dot{x}(t)=f_{1}\left(x_{t}, u(t), t\right), \quad \text { a.e. } t \in\left[t_{0}, t_{1}\right],
$$

where for every $t \in\left[t_{0}, t_{1}\right]$

$$
x_{t}: s \mapsto x(t+s), \quad s \in[-h, 0],
$$

is the past history of $x(\cdot)$ corresponding to the moving time-interval $[t-h, t]$. Throughout the text, $\left[t_{0}, t_{1}\right]$ is a fixed time interval. We suppose that an initial datum $x_{t_{0}}=\varphi$ is given which describes the motion of the system during the time interval $\left[t_{0}-h, t_{0}\right]$. Then, under suitable assumptions on $f_{1}$, the trajectory $x(\cdot)=x_{u}(\cdot)$ of the system is completely determined by the control function $u:\left[t_{0}, t_{1}\right] \rightarrow \mathbb{R}^{r}$. Pertinent existence, uniqueness and continuity results are to be found in [19].

Mathematical models of this type play an important role in every field of science where causes do not produce their effects immediately but with some time delay (see [14] for a brief survey).

Our purpose is to establish a unifying framework in order to derive maximum principles for the following optimum control problems:

(P1) optimal control of HDS towards a given point $x_{1} \in \mathbb{R}^{n}$ :

$$
x\left(t_{1}\right)=x_{1},
$$

(P2) optimal control towards a given target function $\varphi_{1}:[-h, 0] \rightarrow \mathbb{R}^{n}$ :

$$
x_{t_{1}}=\varphi_{1} .
$$

For a discussion of these two problems and a survey of the results obtained as well as the abstract optimization methods employed up to 1973, we refer the reader to [2], [7], [8]. While the abstract variational theory of Neustadt has been efficiently used to derive necessary optimality conditions for problem (P1) (cf. [1], [8]), it encountered severe difficulties, when applied to problem (P2). More recently, some new ideas have been introduced into this context which seem to be quite promising [10], [28] (cf. § 3). The present paper, however, follows the alternative general theory of extremals, the theory of Dubovitskii-Milyutin (cf. [18]). More detailed bibliographical information will be given in the following sections.

\footnotetext{
* Received by the editors November 30, 1976, and in final revised form December 1, 1977.

$\dagger$ Fachbereich Mathematik, Forschungsschwerpunkt Dynamische Systeme, Universität Bremen, 28 Bremen 33, West Germany.
} 
The problems considered jointly in this paper are usually studied separately in the literature. In order to give a comprehensive account we also include some known results or minor generalizations.

In $\S 1$ we establish an abstract local maximum principle that covers the optimal control problems (P1) and (P2). It is shown that the necessary conditions in their nondegenerate form $\left(\lambda_{0}=1\right)$ are also sufficient for optimality in the case of linear systems with convex cost functional. Conditions for nondegeneracy are included in order to clear up the relationship between necessary and sufficient optimality criteria.

A similar maximum principle has been obtained by Kurcyusz [22], as a necessary condition for the optimal control of systems with operator constraints. However, our assumptions are more readily verified. They easily admit the application to HDS with distributed lags and delays in the control, as well as to more general systems described by Fredholm equations.

In $\S 2$ we briefly summarize the results which may be obtained by application of the abstract maximum principle to problem (P1).

In $\S 3$ we apply the results of $\S 1$ to problem (P2), confining ourselves to linear HDS and more generally to Fredholm systems with general control action. We obtain a maximum principle which states necessary and sufficient conditions for optimality. Possible generalizations to nonlinear systems are briefly indicated. Problem (P2) still has been treated by relatively few authors. To our knowledge, all attempts to establish a corresponding maximum principle for general nonlinear functional differential systems have failed up to now (cf. § 3).

Since the maximum principle obtained in $\S 3$ depends essentially on the assumption that the attainability space $\mathscr{A}$ is closed, we investigate this subspace in $\S 4$. Our results generalize some of the theorems obtained in [6]. A conjecture of BanksJacobs-Langenhop $[6$, p. 619] concerning the necessity of their condition (H3) for $\mathscr{A}$ to be closed is partially confirmed (Prop. 4.2). The known criteria for $\mathscr{A}$ to be closed still remain rather unsatisfactory. Only full attainability is completely characterized.

Notation and terminology. Let $X$ be a Banach space and let $X^{*}$ denote its topological dual space. We define the symbol $\left\langle x^{*}, x\right\rangle_{B}$ by $\left\langle x^{*}, x\right\rangle_{B}:=x^{*}(x)$, where the right-hand side is the value of the linear form $x^{*}$ at the point $x$. Let $F: X_{1} \times X_{2} \rightarrow X$ be a map, $X_{1}, X_{2}, X$ B-spaces. Then $D F(x)=D_{1} F\left(x_{1}, x_{2}\right)+D_{2} F\left(x_{1}, x_{2}\right)$ denotes the Fréchet derivative of $F$ in $x=\left(x_{1}, x_{2}\right) \in X_{1} \times X_{2} . \mathscr{L}\left(X_{1}, X_{2}\right)$ is the space of continuous linear operators, mapping $X_{1}$ into $X_{2} . A^{*}$ denotes the adjoint of a continuous linear operator $A$, while $\operatorname{Im} A$ and $\operatorname{Ker} A$ are its range and kernel, respectively; $\operatorname{cl} Q$ is the topological closure of a set $Q$, int $Q$ its interior.

For any subset $Q \subset \mathbb{R}, 1_{Q}$ denotes the characteristic function of $Q$ on $\mathbb{R}$.

1. An abstract local maximum principle. We consider the following abstract control problem (ACP) which reflects the general structure of concrete control problems without presupposing the controls and trajectories to be functions of time:

(ACP) Let $X, U, Z$ be Banach spaces, $Q \subset U, F_{0}: X \times U \rightarrow \mathbb{R}, F_{1}: X \times U \rightarrow X$, $F_{2}: X \rightarrow Z$.

Minimize

subject to

$$
F_{0}(x, u)
$$

$$
\begin{aligned}
& x=F_{1}(x, u), \\
& F_{2}(x)=0, \\
& u \in Q .
\end{aligned}
$$


Later on, $X$ will be interpreted as the space of trajectories, $U$ as the space of control functions, $Q$ as the set of admissible control functions and $F_{2}(x)=0$ as the end condition. $x=F_{1}(x, u)$ corresponds to the equation of motion of the dynamical system.

THEOREM 1.1 (Local maximum principle). Let $\left(x^{0}, u^{0}\right) \in X \times U$ be a solution of (ACP) and suppose the following conditions:

(a) $F_{0}$ is Fréchet-differentiable in $\left(x^{0}, u^{0}\right), F_{1}, F_{2}$ are continuously Fréchetdifferentiable in a neighborhood of $\left(x^{0}, u^{0}\right)$, resp. $x^{0}$.

(b) $D_{1} F_{1}\left(x^{0}, u^{0}\right)$ is a compact operator that satisfies

$$
\operatorname{Ker}\left(\operatorname{Id}_{X}-D_{1} F_{1}\left(x^{0}, u^{0}\right)\right)=0 .
$$

(c) The "attainable subspace" $\mathscr{A}$ of the linearized system

$$
\mathscr{A}:=\left\{D F_{2}\left(x^{0}\right) x \mid \exists u \in U: x=D F_{1}\left(x^{0}, u^{0}\right)(x, u)\right\}
$$

is not a proper dense subspace of $Z$.

(d) $Q$ is convex and contains interior points.

Under these assumptions there exist $\lambda_{0} \geqq 0, x^{*} \in X^{*}, z^{*} \in Z^{*}$, not all zero, which satisfy the following two conditions:

(i) $x^{*}=\lambda_{0} D_{1} F_{0}\left(x^{0}, u^{0}\right)+D_{1} F_{1}\left(x^{0}, u^{0}\right)^{*} x^{*}+D F_{2}\left(x^{0}\right)^{*} z^{*} \quad$ (adjoint equation);

(ii) $\left[\lambda_{0} D_{2} F_{0}\left(x^{0}, u^{0}\right)+D_{2} F_{1}\left(x^{0}, u^{0}\right)^{*} x^{*}\right]\left[u^{0}-u\right] \leqq 0$ for all $u \in Q$

(minimum condition)

Nondegeneracy condition: $\lambda_{0}$ is nonzero if the following additional assumptions are satisfied:

(e) There exist $\tilde{u} \in U, \tilde{x} \in X$ such that $u^{0}+\tilde{u} \in \operatorname{int} Q$ and

$$
\tilde{x}=D F_{1}\left(x^{0}, u^{0}\right)(\tilde{x}, \tilde{u}), \quad 0=D F_{2}\left(x^{0}\right)(\tilde{x}) .
$$

(f) All the points in $Z$ are attainable, that is, $\mathscr{A}=Z$.

Proof. Construct local approximations of the objective function and the constraints by convex cones, and apply the theorem of Dubovitskii-Milyutin in order to establish the generalized equation of Euler-Lagrange for our problem [18]. Then, show that this equation is equivalent to (i) and (ii). For details see [12].

Remark 1.1. In this remark we comment on some of the assumptions of the preceding theorem.

1) Assumption (b) is generally satisfied for ordinary and hereditary differential systems.

2) The most critical assumption of the theorem is (c). It is always satisfied, if we deal with pointwise end conditions ( $Z$ finite dimensional). However, it is difficult to verify, if $Z$ is a function space.

3 ) If the closure of $\mathscr{A}$ is a proper subspace of $Z, \lambda_{0}$ may be chosen to be zero and hence we get necessary conditions which are independent of the objective functional. In this case the theorem tells us something about the "system" (1.1) and its relation to the constraints (1.2), (1.3), but nothing about the optimal control problem: Every solution $\left(x^{0}, u^{0}\right)$ of (1.1)-(1.3), for which the assumptions (a), (b) are met and $\mathrm{cl}(\mathscr{A}) \neq$ $Z$, satisfies conditions (i), (ii) with $\lambda_{0}=0$. The stronger assumption (f) excludes this possibility. It means that the system linearized at $\left(x^{0}, u^{0}\right)$ is completely attainable. This implies that the differential of the function $X \times U \rightarrow X \times Z$ defined by the equality constraints (1.1), (1.2) is surjective. Hence the theorem of Lyusternik may be applied to compute the corresponding tangent cone [12].

4) If both (e) and (f) are satisfied it follows that $\lambda_{0} \neq 0$. We have just seen that only the nondegenerate version of the local maximum principle yields pertinent information for the solution of the optimal control problem. This explains the 
theoretical interest of sufficient conditions for nondegeneracy which should be included in maximum principles whenever possible. One of the strong points of the Dubovitskii-Milyutin method is that, in many cases, it yields conditions for nondegeneracy without much additional effort.

5) Halkin [20] has shown that for finite dimensional terminal conditions (i.e. $Z$ finite dimensional) the assumptions concerning $Q$ and the Fréchet-differentiability of $F_{0}, F_{1}, F_{2}$ can be relaxed.

We now consider the following linear version of the abstract control problem:

(LACP) Let $X, U, Z, Q, F_{0}$ be as before and $A \in \mathscr{L}(X, X), B \in \mathscr{L}(U, X), C \in$ $\mathscr{L}(X, Z), z \in Z$.

Minimize

subject to

$$
F_{0}(x, u)
$$

$$
\begin{aligned}
& x=A x+B u, \\
& C x=z, \\
& u \in Q
\end{aligned}
$$

We shall see that in the linear case the nondegenerate maximum principle is not only a necessary but also a sufficient condition for optimality.

Furthermore, the nondegeneracy assumptions may be weakened. It now suffices to require that the attainability space $\mathscr{A}$ is closed in $Z$ (instead of $\mathscr{A}=Z$ ). To see this, we simply regard the map $u \mapsto F_{2}\left(x_{u}\right)$, where $x_{u}$ is the solution of $x=F_{1}(x, u)$, as a map from $U$ onto $\mathscr{A}$.

THEOREM 1.2. Let $\left(x^{0}, u^{0}\right) \in X \times U$ satisfy the constraints (1.4)-(1.6) and suppose that the following assumptions hold:

(a) $F_{0}$ is Fréchet-differentiable in $\left(x^{0}, u^{0}\right)$ and convex.

(b) $A$ is a compact linear operator satisfying

$$
\operatorname{Ker}\left(\operatorname{Id}_{X}-A\right)=0 \text {. }
$$

(c) $\mathscr{A}=\{C x \mid \exists u \in U: x=A x+B u\}$ is closed in $Z$.

(d) $Q$ is convex and contains interior points.

(e) There exists $(\tilde{x}, \tilde{u}) \in X \times U$ such that

$$
\tilde{x}=A \tilde{x}+B \tilde{u}, \quad C \tilde{x}=z, \quad \tilde{u} \in \operatorname{int} Q .
$$

Then $\left(x^{0}, u^{0}\right)$ is a solution of (LACP) iff the following conditions are satisfied:

There exist $x^{*} \in X^{*}, z^{*} \in Z^{*}$ such that

(i) $x^{*}=D_{1} F_{0}\left(x^{0}, u^{0}\right)+A^{*} x^{*}+C^{*} z^{*}$.

(ii) $\left(D_{2} F_{0}\left(x^{0}, u^{0}\right)+B^{*} x^{*}\right)\left(u^{0}-u\right) \leqq 0$ for all $u \in Q$.

Proof. Let $T: U \rightarrow X$ be the linear mapping which associates with every $u \in U$ the corresponding solution $x_{u}$ of $x=A x+B u$. (LACP) may be reformulated as follows:

$$
\begin{array}{lll}
\left(\mathrm{LACP}^{\prime}\right) & \text { Minimize } & F_{0}(T u, u) \\
& \text { subject to } & (C \circ T) u=z, \quad u \in Q .
\end{array}
$$

$C \circ T$ is a continuous, linear, surjective operator from $U$ onto the Banach space $\mathscr{A}$ (continuity of $T$ follows from Banach's inverse theorem). It is now easy to derive the sufficiency and necessity of conditions (i) and (ii) from the corresponding theorem of Dubovitskii-Milyutin [18, p. 115]. 
Remark 1.2. $G: u \mapsto F_{0}\left(x^{0}, u\right)+x^{*}\left(A x^{0}+B u\right)$ is a convex functional on $Q$. Condition (ii) expresses that the Fréchet derivative of $G$ in $u^{0}$ is a support functional of $Q$ at $u^{0}$. Since $G$ is convex this means that $u^{0}$ is a minimum of $G$ on $Q$. Hence the local minimum condition (ii) is equivalent to the following global minimum condition:

(ii') $F_{0}\left(x^{0}, u^{0}\right)+x^{*}\left(A x^{0}+B u^{0}\right) \leqq F_{0}\left(x^{0}, u\right)+x^{*}\left(A x^{0}+B u\right)$ for all $u \in Q$.

Remark 1.3. With regard to the necessity of (i) and (ii), the central assumption of Theorem 1.2 is (c). However, here it is not needed for calculating the cone of tangent directions (because of linearity, this is a trivial problem). But it is needed in order to determine the dual cone. Kurcyusz [22], [23] has shown the following interesting result: If $\mathscr{A}$ is not closed in $Z$ and $Q=U$, then an objective functional $F_{0}$ exists such that the unique optimal solution of the corresponding problem (LACP) does not satisfy the maximum principle ((i), (ii)).

Remark 1.4. According to Remark 1.1 only the nondegenerate maximum principle can be expected to yield a sufficient criterion for optimality. Assumptions (c) and (e) are used to establish the nondegenerate version of the maximum principle as a necessary criterion. These assumptions become redundant for the sufficiency part of the theorem which presupposes $\lambda_{0}=1$. This corresponds to the well-known fact that Slater's condition is only needed in order to prove the necessity of the saddle point condition for convex programs (see [18, p. 116], [25, p. 216]).

The following reasoning shows that the conditions (c), (e) and int $Q \neq \varnothing$ in (d) can be dispensed with in the proof of sufficiency. Suppose (i) and (ii). Then, by definition of $T$ and the adjoint equation we have for $u \in Q$ :

$$
\left(B^{*} x^{*}\right) u=\left(x^{*} \circ\left(\operatorname{Id}_{X}-A\right) \circ T\right) u=\left(D_{1} F_{0}\left(T u^{0}, u^{0}\right) \circ T\right) u+\left(z^{*} \circ C \circ T\right) u .
$$

This and the analogue equation for $\left(B^{*} x^{*}\right) u^{0}$ show that (ii) implies

$$
\begin{aligned}
\left(D_{2} F_{0}\left(T u^{0}, u^{0}\right)+D_{1} F_{0}\left(T u^{0}, u^{0}\right) \circ T\right)\left(u^{0}-u\right) & \leqq 0 \\
& \text { for all } u \in Q \text { with }(C \circ T) u=z .
\end{aligned}
$$

Since $u \rightarrow F_{0}(T u, u)$ is convex on the convex set $\{u \in Q ;(C \circ T) u=z\}$, we conclude as above that $u^{0}$ is an optimal solution of $\left(\mathrm{LACP}^{\prime}\right)$.

This remark again illustrates the theoretical significance of conditions for nondegeneracy: They specify assumptions under which the maximum principle can be established as a necessary condition in such a form that it becomes sufficient for optimality, if certain convexity conditions are satisfied.

Remark 1.5. Clearly, Theorems 1.1 and 1.2 may also be applied to optimal control problems without end condition. In this case $F_{2} \equiv 0$ and $\mathscr{A}=Z=\{0\}$, assumption (c) is trivially satisfied; and the second Lagrange multiplier $z^{*}$ is zero.

Remark 1.6. Existence of an optimal solution follows, if we assume, e.g., that $U$ is reflexive and $Q$ is closed and bounded. In this case, $Q \cap\{u \in U \mid(C \circ T) u=z\}$ is weakly compact and $F_{0}$ is weakly lower semicontinuous. Unicity of the optimal solution is guaranteed if $F_{0}$ is strictly convex.

\section{Optimal control of hereditary differential systems towards target sets in $\mathbb{R}^{\boldsymbol{n}}$.} Since a large number of papers has been published on this problem (cf. [8]), some of which expose similar optimality conditions, we only briefly indicate the kind of results which can be derived from $\S 1$ :

Consider a nonlinear HDS of type (1) which is to be guided from an initial datum $\varphi$ towards a surface $\left\{x \in \mathbb{R}^{n} ; g(x)=0\right\}, g: \mathbb{R}^{n} \rightarrow \mathbb{R}^{m}$ within a fixed time interval $\left[t_{0}, t_{1}\right]$. Suppose that an integral cost criterion $F_{0}(x, u)=\int_{t_{0}}^{t_{1}} f_{0}(x(t), u(t), t) d t$ and a convex set $\Omega \subset \mathbb{R}^{r}$, int $\Omega \neq \varnothing$ of admissible control values are given. In order to apply the 
theorems of $\S 1$, choose the function spaces $X$ and $U$ of the trajectories and the control functions, respectively, as:

$$
X:=\mathscr{C}\left(\left[t_{0}-h, t_{1}\right], \mathbb{R}^{n}\right) \quad \text { and } \quad U:=L_{\infty}\left(\left[t_{0}, t_{1}\right], \mathbb{R}^{r}\right) .
$$

Then the following results are obtained by translation of Theorems $1.1,1.2$ into the present context:

RESULT 2.1. Under the standard differentiability assumptions for $f_{0}, f_{1}$ and $g, a$ pointwise local maximum principle is derived which generalizes the local maximum principle for ordinary differential systems (cf. [18]).

RESULT 2.2. Nondegeneracy of the maximum principle is established under the following assumptions:

(a) The set of admissible control functions $Q=\{u(\cdot) \in U ; u(t) \in \Omega$ a.e. $\}$ contains in its interior a variation $u^{0}(\cdot)+\tilde{u}(\cdot)$ of the optimal control $u^{0}(\cdot)$ such that the trajectory of the linearized system corresponding to $\tilde{u}(\cdot)$ :

$$
\begin{aligned}
& \dot{\tilde{x}}(t)=D_{1} f_{1}\left(x_{t}^{0}, u^{0}(t), t\right) \tilde{x}_{t}+D_{2} f_{1}\left(x_{t}^{0}, u^{0}(t), t\right) \tilde{u}(t) \text { on }\left[t_{0}, t_{1}\right], \\
& \tilde{x}_{t_{0}} \equiv 0
\end{aligned}
$$

satisfies $D g\left(x^{0}\left(t_{1}\right)\right) \tilde{x}\left(t_{1}\right)=0$.

(b) The linearized system (2.1) is output controllable at time $t_{1}$ in the following sense:

$$
\begin{array}{r}
\forall d \in \mathbb{R}^{m} \exists u \in U \exists x \in X:(x, u) \text { solves }(2.1) \text { and } \\
\\
\operatorname{Dg}\left(x^{0}\left(t_{1}\right)\right)\left(x\left(t_{1}\right)\right)=d .
\end{array}
$$

RESULT 2.3. For linear HDS with convex cost functional and affine end condition it is shown that the nondegenerate maximum principle is a necessary and sufficient optimality criterion, if condition (a) in Result 2.2 is assumed. Furthermore, the maximum principle may be extended to linear HDS with pointwise and distributed lags in the control as in [11].

These results differ only in nuances (e.g. the conditions for nondegeneracy) from those in the literature (e.g., see [1], [3], [11], [27], [31]). The reader interested in explicit formulations and proofs is referred to [12].

However, it should be recognized that the optimal control problem with finite dimensional end condition cannot claim the same importance for HDS as for ordinary differential systems: Problem (P1) neglects the hereditary effects which may force the system to leave the desired value $x\left(t_{1}\right)=x_{1}$ after reaching it. Therefore, in the following section, we shall treat in more detail problem $(\mathrm{P} 2)$ which does conform with the infinite dimensional character of HDS.

3. Optimal control of functional differential systems with function space terminal condition. In this section we consider optimal control problems with linear system equation and function space boundary condition. See Remark 3.2 (below) for the treatment of partially nonlinear systems.

We start with the following problem for HDS which will be generalized later to include systems governed by Fredholm equations.

Problem 3.1. Minimize $\int_{t_{0}}^{t_{1}} f_{0}(x(t), u(t), t) d t$ subject to the constraints:

$$
\begin{array}{ll}
\dot{x}(t)=A(t) x_{t}+B(t) u(t), & t \in\left[t_{0}, t_{1}\right], \\
x(t)=0, & t \in\left[t_{0}-h, t_{0}\right], \\
x\left(t_{1}+s\right)=z(s), & s \in[-h, 0],
\end{array}
$$


where $h \geqq 0, f_{0}: \mathbb{R}^{n} \times \mathbb{R}^{r} \times\left[t_{0}, t_{1}\right] \rightarrow \mathbb{R}, A(t): \mathscr{C}\left([-h, 0], \mathbb{R}^{n}\right) \rightarrow \mathbb{R}^{n}$ is continuous linear, $B(t) \in \mathscr{L}_{n r}$ for $t \in\left[t_{0}, t_{1}\right]$, and $z(\cdot):[-h, 0] \rightarrow \mathbb{R}^{n}$ is a fixed target function.

Following the suggestion of Jacobs-Kao [21], we choose for $Z$ a Sobolev space

$$
Z=W^{1, p}\left([-h, 0], \mathbb{R}^{n}\right), \quad 1 \leqq p \leqq \infty,
$$

where

$$
W^{1, p}\left([\alpha, \beta], \mathbb{R}^{n}\right)=\left\{x \in A C\left([\alpha, \beta], \mathbb{R}^{n}\right) \mid \dot{x} \in L_{p}\right\} .
$$

This is a Banach space with respect to the norm

$$
\|x\|:=|x(\alpha)|+\|\dot{x}\|_{L_{p}} .
$$

The Lagrange multiplier corresponding to the terminal condition is an element of $\left(W^{1, p}\right)^{*}$. While $\left(W^{1, p}\right)^{*} \simeq W^{1, q}$ for $1 \leqq p<\infty, p^{-1}+q^{-1}=1$, the space $\left(W^{1, \infty}\right)^{*}$ cannot be identified with a space of real valued functions (cf. [17, IV, 8.6]). Hence we exclude $p=\infty$.

Kurcyusz-Olbrot [24] analyzed linear systems with constant lag assuming that the coefficient matrices depend analytically on time. Supposing that the control functions are taken from $L_{\tilde{p}}$, they showed that the attainable subspace $\mathscr{A}$ is closed in $W^{1, p}$ $(\tilde{p} \geqq p \geqq 1)$ only if $p=\tilde{p}$. Hence it is not possible to choose $\tilde{p}=\infty$, i.e. $U=$ $L_{\infty}\left(\left[t_{0}, t_{1}\right], \mathbb{R}^{r}\right)$, since we have excluded $p=\infty$. Therefore we shall treat the control problem in the spaces

$$
X:=\underline{W}^{1, p}\left(\left[t_{0}, t_{1}\right], \mathbb{R}^{n}\right), \quad U:=L_{p}\left(\left[t_{0}, t_{1}\right], \mathbb{R}^{r}\right), \quad Z:=W^{1, p}\left([-h, 0], \mathbb{R}^{n}\right)
$$

where ${ }^{1} 2 \leqq p<\infty$ and

$$
W^{1, p}\left(\left[t_{0}, t_{1}\right]_{0} \mathbb{R}^{n}\right):=\left\{x \in W^{1, p}\left(\left[t_{0}-h, t_{1}\right], \mathbb{R}^{n}\right)|x|\left[t_{0}-h, t_{0}\right]=0\right\} .
$$

The choice of $L_{p}$ instead of $L_{\infty}$ entails that pointwise constraints for the controls be excluded since the abstract maximum principle requires that the set of admissible control functions has nonempty interior. $\left\{u \in L_{p} \mid u(t) \in \Omega\right.$ a.e. $\}$ may not have any interior point although int $\Omega \neq \varnothing$ in $\mathbb{R}^{r}$. Therefore we are only allowed to consider control restrictions referring to the whole function, for example energy restrictions of the form $\int_{t_{0}}^{t_{1}}|u(t)|^{2} d t \leqq \alpha(\alpha>0)$.

Let us analyze (3.1) in some more detail. We regard $A(s)$ as a continuous linear map of $X=\underline{W}^{1, p}\left(\left[t_{0}, t_{1}\right], \mathbb{R}^{n}\right)$ into $\mathbb{R}^{n}$ defining

$$
A(s) x:=A(s) x_{s}, \quad x \in X, \quad s \in\left[t_{0}, t_{1}\right] .
$$

Identifying $\left(\underline{W}^{1, p}\right)^{*}$ with $\underline{W}^{1, q}, p^{-1}+q^{-1}=1$, we may assume $A(s) \in \underline{W}^{1, q}\left(\left[t_{0}, t_{1}\right], \mathscr{L}_{n n}\right)$ for all $s \in\left[t_{0}, t_{1}\right]$.

LeMma 3.1. For every $A \in L_{q}\left(\left[t_{0}, t_{1}\right], W^{1, q}\left(\left[t_{0}, t_{1}\right], \mathscr{L}_{n n}\right)\right)$ there is a unique $n \times n$ matrix function $\eta \in L_{q}\left(\left[t_{0}, t_{1}\right] \times\left[t_{0}, t_{1}\right], \mathscr{L}_{n n}\right)$ such that

$$
A(s) x=\int_{t_{0}}^{t_{1}} \eta(s, t) \dot{x}(t) d t, \quad x \in X, \quad s \in\left[t_{0}, t_{1}\right] .
$$

If $A$ is defined by (3.4), $\eta$ satisfies

$$
A(s) x=\int_{t_{0}}^{s} \eta(s, t) \dot{x}(t) d t
$$

\footnotetext{
${ }^{1}$ The condition $p \geqq 2$ is needed to guarantee Fréchet-differentiability of the cost functional.
} 
Proof. The derivation operator $D: \underline{W}^{1, q} \rightarrow L_{q}, D x=\dot{x}$, is a linear isometry of $\underline{W}^{1, q}$ onto $L_{q}$. Let $A_{i}(s) \in W^{1, q}\left(\left[t_{0}, t_{1}\right],\left(\mathbb{R}^{n}\right)^{*}\right)$ be the $i$ th row-vector function of the matrix function $A(s)(i=1, \cdots, n)$. Then $D \circ A_{i} \in L_{q}\left(\left[t_{0}, t_{1}\right], L_{q}\left(\left[t_{0}, t_{1}\right],\left(\mathbb{R}^{n}\right)^{*}\right)\right)$. Reasoning as Dunford-Schwartz [17, III. 11, Lemma 16 and Thm. 17], we obtain uniquely determined functions $\eta_{i} \in L_{q}\left(\left[t_{0}, t_{1}\right] \times\left[t_{0}, t_{1}\right],\left(\mathbb{R}^{n}\right)^{*}\right)(i=1, \cdots, n)$, satisfying $\eta_{i}(s, \cdot)=$ $D \circ A_{i}(s)$ for a.e. $s \in\left[t_{0}, t_{1}\right]$, i.e.

$$
\begin{aligned}
A_{i}(s) x=\left\langle A_{i}(s), x\right\rangle_{W^{p}} & =\left\langle D \circ A_{i}(s), \dot{x}\right\rangle_{L_{\mathrm{p}}}=\left\langle\eta_{i}(s, \cdot), \dot{x}\right\rangle_{L_{\mathrm{p}}} \\
& =\int_{t_{0}}^{t_{1}}\left\langle\eta_{i}(s, t), \dot{x}(t)\right\rangle_{\mathbb{R}^{n}} d t .
\end{aligned}
$$

If we define $\eta(s, t)$ to be the matrix composed of the row vectors $\eta_{i}(s, t)\left(s, t \in\left[t_{0}, t_{1}\right]\right)$, $\eta$ evidently satisfies (3.5). If $A(s) x$ depends only on the values of $x$ on $[s-h, s]$ according to (3.4) it follows from (3.7) that $\eta_{i}(s, t)$ is zero for $t>s$.

The following conclusion is immediate: $x \in X$ is a solution of (3.1) iff $\dot{x}$ is the solution of the following Volterra equation of the second kind [39]:

$$
v(s)=\int_{t_{0}}^{s} \eta(s, t) v(t) d t+B(s) u(s), \quad s \in\left[t_{0}, t_{1}\right]
$$

We see that the HDS (3.1) may be described by a Volterra equation. It seems natural to generalize our analysis to systems described in the same way by Fredholm equations. These Fredholm systems are noncausal (anticipatory), if we continue to interpret $t$ as time. Dynamical systems involving both retarded and advanced effects are employed as models in classical relativistic mechanics [35] and electro-dynamics [16]. Better known is the use of Fredholm equations for the study of two point boundary problems in mathematical physics [13] (e.g. deformation of an elastic rod). In this case $t$ has to be interpreted as a spatial variable and the control is some stationary external force. the form

In the following, we shall deal with Fredholm systems described by an equation of

$$
\dot{x}(s)=A(s) x+y(s), \quad s \in\left[t_{0}, t_{1}\right],
$$

where $y \in L_{p}\left(\left[t_{0}, t_{1}\right], \mathbb{R}^{n}\right)$ and

$$
A \in L_{\infty}\left(\left[t_{0}, t_{1}\right], \underline{W}^{1, q}\left(\left[t_{0}, t_{1}\right], \mathscr{L}_{n n}\right)\right) ;
$$

or equivalently, by the Fredholm equation

$$
\dot{x}(s)=\int_{t_{0}}^{t_{1}} \eta(s, t) \dot{x}(t) d t+y(s), \quad s \in\left[t_{0}, t_{1}\right],
$$

where $\eta$ is uniquely determined by $A$ according to Lemma 3.1. While (3.8) always has a unique solution $v \in L_{p}\left(\left[t_{0}, t_{1}\right], \mathbb{R}^{n}\right)$ for every $u \in L_{p}\left(\left[t_{0}, t_{1}\right], \mathbb{R}^{r}\right)$, we need an additional assumption to assure unique solubility of (3.11).

The linear operator $\hat{A}: L_{p}\left(\left[t_{0}, t_{1}\right], \mathbb{R}^{n}\right) \rightarrow L_{p}\left(\left[t_{0}, t_{1}\right], \mathbb{R}^{n}\right)$, defined by

$$
(\hat{A} v)(s)=\int_{t_{0}}^{t_{1}} \eta(s, t) v(t) d t, \quad s \in\left[t_{0}, t_{1}\right], \quad v \in L_{p},
$$

is compact (cf. [17, VI.9.53]). 
According to Fredholm's alternative, equation (3.11) has a unique solution $\dot{x}(\cdot) \in L_{p}$ for every $y(\cdot) \in L_{p}$ iff the following condition is satisfied:

(3.13) The homogeneous Fredholm equation

$$
v(s)=\int_{t_{0}}^{t_{1}} \eta(s, t) v(t) d t, \quad s \in\left[t_{0}, t_{1}\right]
$$

has only the trivial solution $v \equiv 0$ in $L_{p}\left(\left[t_{0}, t_{1}\right], \mathbb{R}^{n}\right)$.

If we assume (3.13), the Fredholm operator $\operatorname{Id}_{L_{p}}-\hat{A}$ is injective and of index 0 , hence bijective. Therefore the solution operator

$$
(D-\hat{A} \circ D)^{-1}: L_{p}\left(\left[t_{0}, t_{1}\right], \mathbb{R}^{n}\right) \rightarrow \underline{W}^{1, p}\left(\left[t_{0}, t_{1}\right], \mathbb{R}^{n}\right)
$$

of the equation $\dot{x}(s)=A(s) x+y(s), x_{t_{0}} \equiv 0$ is well defined; it is continuous by the open mapping theorem.

We now generalize Problem 3.1 in order to include Fredholm systems with retarded controls:

Problem 3.2. Minimize $\int_{t_{0}}^{t_{1}} f_{0}(x(t), u(t), t) d t$ subject to the constraints

$$
\begin{array}{ll} 
& \dot{x}(t)=A(t) x+\sum_{i=1}^{k} B_{i}(t) u\left(t-h_{i}\right)+\int_{t_{0}}^{t_{1}} B_{0}(t, s) u(s) d s, \quad t \in\left[t_{0}, t_{1}\right], \\
\text { (3.15) } & x \mid\left[a, t_{0}\right]=0, \\
\text { (3.16) } \quad & C\left(x \mid\left[b, t_{1}\right]\right)=z, \\
\text { (3.17) } \quad & u \in Q, \\
\text { where } \quad & a \leqq t_{0} \leqq b \leqq t_{1}, f_{0}: \mathbb{R}^{n} \times \mathbb{R}^{r} \times\left[t_{0}, t_{1}\right] \rightarrow \mathbb{R}, \\
& A \in L_{\infty}\left(\left[t_{0}, t_{1}\right], W^{1, q}\left(\left[t_{0}, t_{1}\right], \mathscr{L}_{n n}\right)\right), \\
& B_{i} \in L_{\infty}\left(\mathbb{R}, \mathscr{L}_{n r}\right), B_{i}=0 \text { outside }\left[t_{0}, t_{1}\right] \text { for } i=1, \cdots, k, \\
& B_{0} \in L_{\infty}\left(\left[t_{0}, t_{1}\right] \times\left[t_{0}, t_{1}\right], \mathscr{L}_{n r}\right), \\
& \left.C \in \mathscr{L}^{1, p}\left(\left[b, W_{1}\right], \mathbb{R}^{n}\right), W^{1, p}\left(\left[b, t_{1}\right], \mathbb{R}^{n}\right)\right), \\
& z \in W^{1, p}\left(\left[b, t_{1}\right], \mathbb{R}^{n}\right), \\
& Q \subset L_{p}\left(\left[t_{0}, t_{1}\right], \mathbb{R}^{r}\right) \text { are fixed. }
\end{array}
$$

We assume $u=0$ outside $\left[t_{0}, t_{1}\right]$.

The following theorem is obtained by application of Theorem 1.2 to Problem 3.2.

THEOREM 3.1. Let $\left(x^{0}, u^{0}\right) \in W^{1, p}\left(\left[a, t_{1}\right], \mathbb{R}^{n}\right) \times L_{p}\left(\left[t_{0}, t_{1}\right], \mathbb{R}^{r}\right)$ satisfy the constraints of Problem 3.2 and assume the following conditions:

(a) $f_{0}(x, u, t)$ is continuously Fréchet-differentiable and convex with respect to $(x, u)$, measurable in $t$ and for every $K>0$ there exist $m_{1}(\cdot) \in L_{1}\left(\left[t_{0}, t_{1}\right], \mathbb{R}\right)$, $m_{2} \in \mathbb{R}_{+}, m_{3}(\cdot) \in L_{q}\left(\left[t_{0}, t_{1}\right], \mathbb{R}\right)$ such that

$$
\begin{aligned}
& \left|f_{0}(x, u, t)\right|+\left|D_{1} f_{0}(x, u, t)\right| \leqq m_{1}(t)+m_{2}|u|^{p}, \\
& \left|D_{2} f_{0}(x, u, t)\right| \leqq m_{3}(t)+m_{2}|u|^{p-1}
\end{aligned}
$$

for all $x \in \mathbb{R}^{n},|x| \leqq K$, all $u \in \mathbb{R}^{r}$ and a.e. $t \in\left[t_{0}, t_{1}\right]$.

(b) Condition (3.13) is satisfied with $\eta$ defined as in Lemma 3.1.

(c) $\mathscr{A}:=\left\{C\left(x \mid\left[b, t_{1}\right]\right) \mid \exists u \in L_{p}\left(\left[t_{0}, t_{1}\right], \mathbb{R}^{r}\right):(x, u)\right.$ satisfies $\left.(3.15)\right\}$ is a closed linear subspace of $W^{1, p}\left(\left[b, t_{1}\right], \mathbb{R}^{n}\right)$.

(d) $Q \subset L_{p}\left(\left[t_{0}, t_{1}\right], \mathbb{R}^{r}\right)$ is convex and has nonempty interior.

(e) There is $(\tilde{x}, \tilde{u}) \in W^{1, p}\left(\left[a, t_{1}\right], \mathbb{R}^{n}\right) \times L_{p}\left(\left[t_{0}, t_{1}\right], \mathbb{R}^{r}\right)$ satisfying (3.15), (3.16) and $\tilde{u} \in$ int $Q$.

(f) $\operatorname{Im} C$ is closed. 
Then $\left(x^{0}, u^{0}\right)$ is optimal iff there exist $\psi, \rho \in L_{q}\left(\left[t_{0}, t_{1}\right], \mathbb{R}^{n}\right), p^{-1}+q^{-1}=1, \rho \mid\left[t_{0}, b\right]$ constant such that

$$
\begin{gathered}
\psi(t)=\int_{t}^{t_{1}} D_{1} f_{0}\left(x^{0}(s), u^{0}(s), s\right) d s+\int_{t_{0}}^{t_{1}} \eta(s, t)^{*} \psi(s) d s+\rho(t) \\
\int_{t_{0}}^{t_{1}}\left\langle D_{2} f_{0}\left(x^{0}(t), u^{0}(t), t\right)+\sum_{i=1}^{k} B_{i}\left(t+h_{i}\right)^{*} \psi\left(t+h_{i}\right)\right. \\
\left.+\int_{t_{0}}^{t_{1}} B_{0}(s, t)^{*} \psi(s) d s, u^{0}(t)-u(t)\right\rangle_{\mathbb{R}^{r}} d t \leqq 0 \quad \text { for all } u \in Q .
\end{gathered}
$$

(3.20) The function $\rho(b)+\int_{b}^{t} \rho(s) d s, t \in\left[b, t_{1}\right]$, is orthogonal to $\operatorname{Ker} C$.

Proof. Let $X:=\underline{W}^{1, p}\left(\left[t_{0}, t_{1}\right], \mathbb{R}^{n}\right), U:=L_{p}\left(\left[t_{0}, t_{1}\right], \mathbb{R}^{r}\right), Z:=W^{1, p}\left(\left[b, t_{1}\right], \mathbb{R}^{n}\right)$, and define

$$
\begin{aligned}
& F_{0}(x, u)=\int_{t_{0}}^{t_{1}} f_{0}(x(t), u(t), t) d t \\
& (\tilde{A} x)(t):=\int_{t_{0}}^{t} A(s) x d s, \quad t \in\left[t_{0}, t_{1}\right] \\
& (\tilde{B} u)(t):=\int_{t_{0}}^{t}\left[\sum_{i=1}^{k} B_{i}(s) u\left(s-h_{i}\right)+\int_{t_{0}}^{t_{1}} B_{0}(s, \tau) u(\tau) d \tau\right] d s, \quad t \in\left[t_{0}, t_{1}\right], \\
& \tilde{C} x:=C\left(x \mid\left[b, t_{1}\right]\right)
\end{aligned}
$$

for $x \in X, u \in U$.

Then Problem 3.2 is equivalent to

$\begin{array}{ll}\text { Minimize } & F_{0}(x, u) \text { on } X \times U \\ \text { subject to } & x=\tilde{A} x+\tilde{B} u, \\ & \tilde{C} x=z, \\ & u \in Q .\end{array}$

Using (a) we have to prove that $F_{0}$ is well-defined and Fréchet-differentiable in $(x, u)$. By use of Lebesgue's theorem on dominated convergence, it may be shown that $F_{0}$ is continuously differentiable in $x$. It remains to prove continuous differentiability in $u$. This is much more difficult (see [34, Thm. 21.1]) and requires application of a theorem of Gavurin. The operators $\tilde{A}: X \rightarrow X, \tilde{B}: U \rightarrow X$ and $\tilde{C}: X \rightarrow Z$ are well-defined, continuous and linear. $\tilde{A}=D^{-1} \circ \hat{A} \circ D$ is a compact linear operator on $W^{1, p}$ (cf. (3.12)) satisfying $\operatorname{Ker}\left(\operatorname{Id}_{W^{1, p}}-\tilde{A}\right)=0$ by (b).

The other conditions of Theorem 1.2 follow immediately from (c)-(e).

Thus $\left(x^{0}, u^{0}\right)$ is a solution of Problem 3.2 iff there are $x^{*} \in X^{*}, z^{*} \in Z^{*}$ with

$$
\begin{aligned}
& x^{*}=D_{1} F_{0}\left(x^{0}, u^{0}\right)+\tilde{A}^{*} x^{*}+\tilde{C}^{*} z^{*} \\
& {\left[D_{2} F_{0}\left(x^{0}, u^{0}\right)+\tilde{B}^{*} x^{*}\right]\left(u^{0}-u\right) \leqq 0 \text { for all } u \in Q}
\end{aligned}
$$

$\left(\underline{W}^{1, p}\right)^{*}$ and $\left(L^{p}\right)^{*}$ are identified with $\underline{W}^{1, q}$ and $L^{q}$, respectively $\left(p^{-1}+q^{-1}=1\right)$. 
Computation of the operators in (3.21) and (3.22) yields

$$
\begin{gathered}
D_{1} F_{0}\left(x^{0}, u^{0}\right)(t)=\int_{t_{0}}^{t} \int_{\tau}^{t_{1}} D_{1} f_{0}\left(x^{0}(s), u^{0}(s), s\right) d s d \tau, \quad t \in\left[t_{0}, t_{1}\right] ; \\
\left(\tilde{A}^{*} x^{*}\right)(t)=\int_{t_{0}}^{t} \int_{t_{0}}^{t_{1}} \eta(s, \tau)^{*} x^{*}(s) d s d \tau, \quad t \in\left[t_{0}, t_{1}\right] ; \\
\left(\tilde{C}^{*} z^{*}\right)(t)= \begin{cases}\left(t-t_{0}\right)\left(C^{*} z^{*}\right)(b), & t \in\left[t_{0}, b\right], \\
\left(b-t_{0}-1\right)\left(C^{*} z^{*}\right)(b)+\left(C^{*} z^{*}\right)(t), & t \in\left[b, t_{1}\right] ;\end{cases} \\
D_{2} F_{0}\left(x^{0}, u^{0}\right)(t)=D_{2} f_{0}\left(x^{0}(t), u^{0}(t), t\right), \quad t \in\left[t_{0}, t_{1}\right] ; \\
\left(\tilde{B}^{*} x^{*}\right)(t)=\sum_{i=1}^{k} B_{i}\left(t+h_{i}\right)^{*} \frac{d x^{*}}{d t}\left(t+h_{i}\right)+\int_{t_{0}}^{t_{1}} B_{0}(s, t)^{*} \frac{d x^{*}}{d s}(s) d s, \quad t \in\left[t_{0}, t_{1}\right] .
\end{gathered}
$$

Define

$$
\begin{aligned}
& \psi(t):=\frac{d}{d t} x^{*}(t), \quad t \in\left[t_{0}, t_{1}\right] \\
& \rho(t):=\frac{d}{d t}\left(\tilde{C}^{*} z^{*}\right)(t)= \begin{cases}\left(C^{*} z^{*}\right)(b), & t \in\left[t_{0}, b\right], \\
\frac{d}{d t}\left(C^{*} z^{*}\right)(t), & t \in\left(b, t_{1}\right] .\end{cases}
\end{aligned}
$$

Then (3.21) and (3.22) yield (3.18) and (3.19). The transversality condition (3.20) is a consequence of the definition of $\tilde{C}$ and $\rho$.

Conversely (3.18)-(3.20) imply the existence of $x^{*}, z^{*}$ satisfying (3.21) and (3.22), because by (f): $\operatorname{Im} C^{*}=(\operatorname{Ker} C)^{\perp}$.

Remark 3.1. Convexity of $f_{0}$ and condition (f) are needed only for sufficiency, while conditions (c), (e) and int $Q \neq \varnothing$ are needed only for necessity.

Remark 3.2. It is possible to generalize Theorem 3.1 to nonlinear systems by application of the abstract maximum principle (Theorem 1.1); but the control $u$ must appear linearly in the system's equation to ensure Fréchet-differentiability of $F_{1}$ (see [22], [33]). In fact, suppose the system's equation is given as in (1) by

$$
\dot{x}(t)=f_{1}\left(x_{t}, u(t), t\right), \quad t \in\left[t_{0}, t_{1}\right] .
$$

If $F_{1}: \underline{W}^{1, p} \times L_{p} \rightarrow \underline{W}^{1, p}$, defined by $F_{1}(x, u)(t)=\int_{t_{0}}^{t} f_{1}\left(x_{\tau}, u(\tau), \tau\right) d \tau$, is Fréchetdifferentiable, then for any fixed $x(\cdot) \in \underline{W}^{1, p}$ the map

$$
\begin{aligned}
& u \mapsto\left(t \mapsto \int_{t_{0}}^{t} f_{1}\left(x_{\tau}, u(\tau), \tau\right) d \tau\right) \\
& L_{p}\left(\left[t_{0}, t_{1}\right], \mathbb{R}^{r}\right) \rightarrow \underline{W}^{1, p}\left(\left[t_{0}, t_{1}\right), \mathbb{R}^{n}\right)
\end{aligned}
$$

and hence the map

$$
\begin{aligned}
& u \mapsto\left(t \mapsto f_{1}\left(x_{t}, u(t), t\right)\right) \\
& L_{p}\left(\left[t_{0}, t_{1}\right], \mathbb{R}^{r}\right) \rightarrow L_{p}\left(\left[t_{0}, t_{1}\right], \mathbb{R}^{n}\right)
\end{aligned}
$$

must be Fréchet-differentiable.

Now the latter mapping is a superposition operator for which Vainberg [33, pp. 90-91] has proved (for $p=2, r=n=1$ ) the following: 
LemMA. Suppose $G: L_{p}\left(\left[t_{0}, t_{1}\right], \mathbb{R}^{r}\right) \rightarrow L_{p}\left(\left[t_{0}, t_{1}\right], \mathbb{R}^{n}\right)$ is given by

$$
G(u)(t)=g(u(t), t), \text { a.e. } t \in\left[t_{0}, t_{1}\right]
$$

where $g: \mathbb{R}^{r} \times\left[t_{0}, t_{1}\right] \rightarrow \mathbb{R}^{n}$. Then $G$ is Fréchet-differentiable iff $g(\cdot, t)$ is affine for a.e. $t \in\left[t_{0}, t_{1}\right]$.

Thus $F_{1}$ may be expected to be Fréchet-differentiable only if it is linear in $u$. Therefore, necessary conditions analogous to Theorem 3.1 may be derived from Theorem 1.1 only for Fredholm systems described by equations of the following form (neglecting delays in the control):

$$
\dot{x}(t)=f(x, t)+g(x, t) u(t)
$$

where $f: W^{1, p} \times\left[t_{0}, t_{1}\right] \rightarrow \mathbb{R}^{n}, g: W^{1, p} \times\left[t_{0}, t_{1}\right] \rightarrow \mathscr{L}_{n r}$.

Conditions on $f, g$ which are sufficient for $F_{1}$ to be continuously Fréchetdifferentiable can be found in [22], [40].

Remark 3.3. Comparison of Theorem 3.1 with the maximum principle known for $\mathbb{R}^{n}$-targets (cf. $\S 2$ ) shows the following differences:

-in Theorem 3.1 the minimum condition can be established only in integral form as distinguished from the usual pointwise form;

-in the case of $\mathbb{R}^{n}$-targets, the solution $\psi$ of the adjoint equation is known to be of bounded variation on $\left[t_{0}, t_{1}\right]$, left continuous on $\left(t_{0}, t_{1}\right]$, continuous at $t_{0}$. The corresponding function $\psi$ in Theorem 3.1 is only in $L^{q}$. But suppose that for $s \in\left[t_{0}, t_{1}\right], A(s): W^{1, p}\left(\left[t_{0}, t_{1}\right], \mathbb{R}^{n}\right) \rightarrow \mathbb{R}^{n}$ can be extended to a continuous linear function on $\mathscr{C}\left(\left[t_{0}, t_{1}\right], \mathbb{R}^{n}\right)$. Then $\eta(s, \cdot)$ is of bounded variation and thus, since $\rho \mid\left[t_{0}, b\right]$ is constant, $\psi$ is of bounded variation on $\left[t_{0}, b\right]$.

Remark 3.4. For HDS the adjoint equation (3.18) has the form

$$
\psi(t)=\int_{t}^{t_{1}} D_{1} f_{0}\left(x^{0}(s), u^{0}(s), s\right) d s+\int_{t}^{t_{1}} \eta(s, t)^{*} \psi(s) d s+\rho(t)
$$

because $\eta(s, t)=0$ for $s<t$.

Problem 3.2 includes also problems with lagged controls, where $h_{i} \geqq 0$ for $i=$ $1, \cdots, k$ and $B_{0} \equiv 0$.

Remark 3.5. The terminal condition $C\left(x \mid\left[b, t_{1}\right]\right)=z$ is rather flexible and includes the following cases:

1) $C=0$ and $z=0$ : no terminal condition.

2) $b=t_{1}$ : finite dimensional terminal condition as in $\S 2$.

$3)$ suppose, it is required that $x$ vanishes in $l$ fixed time points $t^{(i)} \in\left[b, t_{1}\right]$, $i=1, \cdots, l$. Let $p=2$, so $Z=W^{1,2}$ is a Hilbert space, and define

$$
V=\left\{y \in W^{1,2}\left(\left[b, t_{1}\right], \mathbb{R}^{n}\right): y\left(t^{(i)}\right)=0, i=1, \cdots, l\right\} .
$$

$V$ is a closed subspace of $W^{1,2}$ with finite dimensional orthogonal complement $V^{\perp}$. If we take $C$ as the projection of $Z$ onto $V^{\perp}$, the desired terminal condition is described by

$$
C\left(x \mid\left[b, t_{1}\right]\right)=0 .
$$

4) $C=\operatorname{Id}_{W^{1, p}}\left(\left[b, t_{1}\right], \mathbb{R}^{n}\right)$ : fixed target function as in Problem 3.1 .

In the first three cases $\mathscr{A}$ is clearly a closed subspace of $W^{1, p}$. In the fourth case $\mathscr{A}$ is closed only under additional assumptions which will be studied later.

The work we know which has been done on control problems with function space terminal conditions is restricted to HDS, which are sometimes allowed to be of neutral type, i.e. have delays in $\dot{x}$, too. 
It is easy to show that the adjoint equation (3.18) coincides with those in [3], [4], [7], [10], [21], [22] for the corresponding classes of HDS (cf. [8]). We remark that the maximum principle in [21] is true only under the additional assumption that the control $u$ appears linearly in the system's equation (cf. Remark 3.2 above).

The necessary conditions derived by Banks-Kent [7] for quite general neutral HDS are not totally satisfactory, because nontriviality is not guaranteed; however, assuming nondegeneracy, they established sufficiency. Theorem 3.1 gives conditions, which imply nondegeneracy. Bien [10] has tried to solve Problem 3.1 by transforming the functional end condition into a mixed control-phase variable equality constraint plus a finite dimensional end condition. He then applied the Neustadt-Makowski theory [26]. But in order to get Lagrange multipliers, which can be identified with functions on $\left[t_{0}, t_{1}\right]$, a rather strong regularity condition is needed.

For a discussion of results on optimal control problems involving integral equations of Volterra type compare [8], [27]. See also [36], [37], resp. [38], for a treatment of dynamical systems described by functional integral equations and Fredholm integral equations. In [38], it is shown that under additional assumptions, it is not necessary to assume unique solubility (3.13). However, all these papers on integral equations only apply to problems with finite dimensional end conditions.

The crucial assumption of our Theorem 3.1 is (c): $\mathscr{A}$ has to be closed in the infinite-dimensional B-space $Z=W^{1, p}\left(\left[b, t_{1}\right], \mathbb{R}^{n}\right)$. The counterexample (cf. $\left.\S 1\right)$ of Kurcyusz [22] shows that this assumption may not be weakened. If $\mathscr{A}$ is not closed (resp. dense and not closed), a quadratic real function $f_{0}$ exists, so that the unique solution of the corresponding optimal control problem, Problem 3.2, does not satisfy the nondegenerate maximum principle (3.18)-(3.20) (resp. only satisfies the trivial form of the maximum principle with $\left.\lambda_{0}=0, \psi \equiv 0, \rho \equiv 0\right)$.

The next section will be concerned with the problem of finding necessary and-or sufficient conditions for $\mathscr{A}$ to be closed or to equal $Z$. The equality $\mathscr{A}=Z$ which represents a very restrictive attainability condition (Proposition 4.3 below), is necessary in order to derive a nondegenerated maximum principle for nonlinear HDS (see Theorem 1.1).

4. Analysis of the attainable subspace. We exclude time-delays of the control function and consider the system

$$
\begin{aligned}
& \dot{x}(t)=A(t) x+B(t) u(t), \quad t \in\left[t_{0}, t_{1}\right], \\
& x \mid\left[a, t_{0}\right]=0
\end{aligned}
$$

where $u \in U=L^{p}\left(\left[t_{0}, t_{1}\right], \mathbb{R}^{r}\right), 1<p<\infty, a \leqq t_{0}<b \leqq t_{1}$.

In order to simplify the analysis, we assume

$$
\mathscr{A}=\left\{x\left|\left[b, t_{1}\right]\right| \exists u \in U,(x, u)\right. \text { satisfies (4.1)\}, }
$$

that is, we regard the problem of control towards a fixed target function in $Z$. We presuppose (3.10), (3.13) and $B \in L_{\infty}\left(\left[t_{0}, t_{1}\right], \mathscr{L}_{n r}\right)$. Hence (4.1) has a unique solution for each $u \in U$.

We decompose the operator $A(s), s \in\left[t_{0}, t_{1}\right]$, into two additive components (cf. Lemma 3.1)

$$
\begin{array}{ll}
A^{1}(s): \underline{W}^{1, p}\left(\left[t_{0}, b\right], \mathbb{R}^{n}\right) \rightarrow \mathbb{R}^{n}, \quad A^{1}(s) x=\int_{t_{0}}^{b} \eta(s, t) \dot{x}(t) d t, \\
A^{2}(s): W^{1, p}\left(\left[b, t_{1}\right], \mathbb{R}^{n}\right) \rightarrow \mathbb{R}^{n}, \quad A^{2}(s) x=\int_{b}^{t_{1}} \eta(s, t) \dot{x}(t) d t .
\end{array}
$$

Then $A(s) x=A^{1}(s)\left(x \mid\left[t_{0}, b\right]\right)+A^{2}(s)\left(x \mid\left[b, t_{1}\right]\right)$, for $x \in X=W^{1, p}\left(\left[t_{0}, t_{1}\right], \mathbb{R}^{n}\right)$. 
Throughout the rest of this section we assume that $A^{1}, B$ satisfy the following two conditions:

$$
\left(t \rightarrow\left\|B(t)^{+} A^{1}(t)\right\|\right) \in L_{p}\left(\left[b, t_{1}\right], \mathbb{R}\right),
$$

where $B(t)^{+}$is the generalized inverse of the matrix $B(t)$ (Penrose [30]) and

$$
\left\|B(t)^{+} A^{1}(t)\right\|:=\sup \left\{\mid B(t)^{+} A^{1}(t) x\left\|x \in \underline{W}^{1, p},\right\| x \| \leqq 1\right\} .
$$

(4.3) The equation

$$
\dot{x}(s)=A^{1}(s) x\left|\left[t_{0}, b\right]+w(s), \quad s \in\left[t_{0}, b\right], \quad x\right|\left[a, t_{0}\right]=0
$$

has a unique solution $x$ for each $w \in L_{p}\left(\left[t_{0}, b\right], \mathbb{R}^{n}\right)$.

The following hypotheses will be referred to in the sequel:

(H1) For all $d \in \mathbb{R}^{n}$ there is $v \in L_{p}\left(\left[t_{0}, b\right], \mathbb{R}^{r}\right)$ such that the solution $y$ of

$$
y \mid\left[a, t_{0}\right]=0, \quad \dot{y}(t)=A^{1}(t) y+B(t) v(t), \quad t \in\left[t_{0}, b\right],
$$

satisfies

$$
y(b)=d
$$

(H2) $\operatorname{Im} A^{1}(t) \subset \operatorname{Im} B(t)$ for a.e. $t \in\left[b, t_{1}\right]$.

(H3) $B(t)^{+}$is bounded a.e. on $\left[b, t_{1}\right]$.

(H4) Rank $B(t)=n$ for a.e. $t \in\left[b, t_{1}\right]$.

Some comments on these hypotheses are appropriate: $(\mathrm{H} 1)$ is equivalent to complete pointwise attainability at time $b$ for HDS. (H2) is equivalent to

$$
A^{1}(t) x=B(t) B(t)^{+} A^{1}(t) x \quad \text { for all } x \in W^{1, p}\left(\left[t_{0}, b\right], \mathbb{R}^{n}\right), \quad \text { a.e. } t \in\left[b, t_{1}\right],
$$

i.e. $A^{1}(t)$ factors through $B(t)$ for a.e. $t$.

Condition (4.2) implies that

$$
\left(t \mapsto B(t)^{+} A^{1}(t) x\right) \in L_{p}\left(\left[b, t_{1}\right], \mathbb{R}^{r}\right) .
$$

Thus ( $\mathrm{H} 2)$ admits the following intuitive interpretation: The hereditary effects on $\dot{x} \mid\left[b, t_{1}\right]$, produced by the values $x(s), s \in\left[t_{0}, b\right]$ via $A^{1}$, can be compensated by suitable control functions in $L_{p}$.

If $B(\cdot)$ is continuous, Kurcyusz-Olbrot [24] have shown that (H3) is satisfied iff Rank $B(t)$ is constant on $\left[b, t_{1}\right]$.

$(\mathrm{H} 4)$ is a very strong condition, requiring in particular that the dimension $r$ of the control space is not less than the dimension $n$ of the phase space. Evidently ( $\left.\mathrm{H}_{4}\right)$ implies (H2).

For HDS, (H1)-(H3) correspond to the hypotheses $(\mathrm{H} 1)-(\mathrm{H} 3)$ in [6], letting $b=t_{1}-h$.

Proposition 4.1. If the hypotheses $(\mathrm{H} 1),(\mathrm{H} 4)$ are valid, $\mathscr{A}$ is dense in $W^{1, p}\left(\left[b, t_{1}\right], \mathbb{R}^{n}\right)$. Conversely, if $\mathscr{A}$ is dense in $W^{1, p}\left(\left[b, t_{1}\right], \mathbb{R}^{n}\right),(\mathrm{H} 1)$ must be valid.

Proof. If $\mathscr{A}$ is dense in $W^{1, p}\left(\left[b, t_{1}\right], \mathbb{R}^{n}\right)$ and $d \in \mathbb{R}^{n}$, a sequence $\left(u_{k}\right)$ exists in $L_{p}\left(\left[t_{0}, t_{1}\right], \mathbb{R}^{r}\right)$ such that the corresponding solutions $\left(x_{k}\right)$ of $(4.1)$ converge to the constant function $z(t) \equiv d$ in $W^{1, p}\left(\left[b, t_{1}\right], \mathbb{R}^{n}\right)$. Hence by $(3.10)$

$$
x_{k}(b) \rightarrow d \quad \text { and } \quad A^{2}(t) x_{k} \mid\left[b, t_{1}\right]=\int_{b}^{t_{1}} \eta(t, s) \dot{x}_{k}(s) d s \rightarrow 0
$$


uniformly in $t \in\left[b, t_{1}\right]$, if $k \rightarrow \infty$. Let $T$ be the solution operator of the equation in (4.3). Then

$$
x_{k} \mid\left[t_{0}, b\right]=T\left(A^{2}(\cdot) x_{k} \mid\left[b, t_{1}\right]+B(\cdot) u_{k}(\cdot)\right) .
$$

Since $T$ is continuous (cf. (3.14)), we obtain for $z_{k}:=T\left(B(\cdot) u_{k}(\cdot)\right)$ by (4.5)

$$
\left\|z_{k}-x_{k} \mid\left[t_{0}, b\right]\right\|=\left\|T\left(A^{2}(\cdot) x_{k} \mid\left[b, t_{1}\right]\right)\right\| \rightarrow 0
$$

where the norm is taken in $W^{1, p}\left(\left[t_{0}, b\right], \mathbb{R}^{n}\right)$.

Hence $\lim _{k \rightarrow \infty} z_{k}(b)=d$. (H1) follows, because the only dense linear subspace of $\mathbb{R}^{n}$ is the whole space.

Now assume (H1) and (H4) and let $z \in W^{1, p}\left(\left[b, t_{1}\right], \mathbb{R}^{n}\right)$. We shall construct a sequence $\left(x_{k}, u_{k}\right) \in W^{1, p}\left(\left[t_{0}, t_{1}\right], \mathbb{R}^{n}\right) \times L_{p}\left(\left[t_{0}, t_{1}\right], \mathbb{R}^{r}\right)$, satisfying the system's equation such that $\left(x_{k} \mid\left[b, t_{1}\right]\right)$ converges to $z$.

Condition (4.3) implies the existence of $\tilde{y}$ with

$$
\tilde{y} \mid\left[a, t_{0}\right]=0, \quad \dot{y}(s)=A^{1}(s) \tilde{y}+A^{2}(s) z, \quad s \in\left[t_{0}, b\right] .
$$

By (H1) there are $\hat{y}, v$ with

$$
\hat{y} \mid\left[a, t_{0}\right]=0, \quad \hat{y}(s)=A^{1}(s) \hat{y}+B(s) v(s), \quad s \in\left[t_{0}, b\right],
$$

and

$$
\hat{y}(b)=z(b)-\tilde{y}(b) .
$$

Define $y=\hat{y}+\tilde{y}$. Then

$$
y \mid\left[a, t_{0}\right]=0, \quad \dot{y}(s)=A^{1}(s) y+B(s) v(s)+A^{2}(s) z, \quad s \in\left[t_{0}, b\right]
$$

and $y(b)=z(b)$.

Define $x \in X:=\underline{W}^{1, p}\left(\left[t_{0}, t_{1}\right], \mathbb{R}^{n}\right)$ by

$$
x(s)= \begin{cases}y(s) & \text { for } s \in[a, b] \\ z(s) & \text { for } s \in\left[b, t_{1}\right]\end{cases}
$$

and a measurable, not necessarily integrable function $w$ by

$$
w(s)= \begin{cases}v(s), & s \in\left[t_{0}, b\right), \\ B(s)^{*}\left[B(s) B(s)^{*}\right]^{-1}[\dot{x}(s)-A(s) x], & s \in\left[b, t_{1}\right] .\end{cases}
$$

$w$ is a.e. defined by condition (H4). By definition we get $B(\cdot) w(\cdot) \in L_{p}\left(\left[t_{0}, t_{1}\right], \mathbb{R}^{n}\right)$ and

$$
\dot{x}(s)=A(s) x+B(s) w(s), \quad s \in\left[t_{0}, t_{1}\right] .
$$

Let $M_{k}:=\left\{s \in\left[t_{0}, t_{1}\right]:|w(s)|<k\right\}$ and

$$
w_{k}(s):= \begin{cases}w(s) & \text { for } s \in M_{k}, \\ 0 & \text { for } s \in\left[t_{0}, t_{1}\right] \backslash M_{k} .\end{cases}
$$

Then $w_{k} \in L_{p}\left(\left[t_{0}, t_{1}\right], \mathbb{R}^{r}\right)$ and $B(\cdot) w_{k}(\cdot)$ converges to $B(\cdot) w(\cdot)$ in $L_{p}\left(\left[t_{0}, t_{1}\right], \mathbb{R}^{n}\right)$ if $k \rightarrow \infty$.

The solutions $x_{k} \in X$ of

$$
x_{k} \mid\left[a, t_{0}\right]=0, \quad \dot{x}_{k}(s)=A(s) x_{k}+B(s) w_{k}(s), \quad s \in\left[t_{0}, t_{1}\right],
$$

converge to $x$ in $X$, since the solution operator of this equation is continuous by (3.14). Hence the sequence $\left(x_{k} \mid\left[b, t_{1}\right]\right)$ converges to $z$ in $W^{1, p}\left(\left[b, t_{1}\right], \mathbb{R}^{n}\right)$. 
Proposition 4.2. Let $(\mathrm{H} 2)$ be satisfied. Then $\mathscr{A}$ is closed in $W^{1, p}\left(\left[b, t_{1}\right], \mathbb{R}^{n}\right)$ iff (H3) is valid.

Proof. We define the continuous multiplication operator $\tilde{B}$ from $L_{p}\left(\left[b, t_{1}\right], \mathbb{R}^{r}\right)$ to $L_{p}\left(\left[b, t_{1}\right], \mathbb{R}^{n}\right)$ by

$$
(\tilde{B} u)(t):=B(t) u(t), \quad t \in\left[b, t_{1}\right] .
$$

Kurcyusz-Olbrot [24] have shown that $(\mathrm{H} 3)$ is satisfied (i.e. $\left.B(\cdot)^{+} \in L_{\infty}\left(\left[b, t_{1}\right], \mathscr{L}_{m}\right)\right)$, iff $\operatorname{Im} \tilde{B}$ is closed. Suppose that $(\mathrm{H} 3)$ is not valid. Then there exists a function $w \in \operatorname{cl}(\operatorname{Im} \tilde{B}) \backslash \operatorname{Im} \tilde{B}$. Define $x$ as solution of

$$
x \mid\left[a, t_{0}\right]=0, \cdot \dot{x}(t)=A(t) x+ \begin{cases}0, & t \in\left[t_{0}, b\right), \\ w(t), & t \in\left[b, t_{1}\right] .\end{cases}
$$

Since $w \in \operatorname{cl}(\operatorname{Im} \tilde{B})$ and the solution operator is continuous by (3.14), we get $x \mid\left[b, t_{1}\right] \in$ cl $\mathscr{A}$. Let us suppose for a moment that $x \mid\left[b, t_{1}\right] \in \mathscr{A}$. In this case there is a pair $(y, u) \in X \times U$ with

$$
y \mid\left[a, t_{0}\right]=0, \quad \dot{y}(t)=A(t) y+B(t) u(t), \quad t \in\left[t_{0}, t_{1}\right],
$$

and $y\left|\left[b, t_{1}\right]=x\right|\left[b, t_{1}\right]$. Then a.e. on $\left[b, t_{1}\right]$,

$$
\begin{aligned}
A^{1}(t) x\left|\left[t_{0}, b\right]+A^{2}(t) x\right|\left[b, t_{1}\right]+w(t)=\dot{x}(t) & =\dot{y}(t) \\
& =A^{1}(t) y\left|\left[t_{0}, b\right]+A^{2}(t) y\right|\left[b, t_{1}\right]+B(t) u(t) .
\end{aligned}
$$

By (4.4), we obtain for a.e. $t \in\left[b, t_{1}\right]$ :

$$
\begin{aligned}
w(t) & =A^{1}(t)(y-x) \mid\left[t_{0}, b\right]+B(t) u(t) \\
& =B(t)\left[B(t)^{+} A^{1}(t)(y-x) \mid\left[t_{0}, b\right]+u(t)\right] .
\end{aligned}
$$

Condition (4.2) implies that

$$
B(\cdot)^{+} A^{1}(\cdot)(y-x) \mid\left[t_{0}, b\right] \in L_{p}\left(\left[b, t_{1}\right], \mathbb{R}^{r}\right),
$$

and thus $w \in \operatorname{Im} \tilde{B}$. This contradiction shows that $x \mid\left[b, t_{1}\right] \in \operatorname{cl}(\mathscr{A}) \backslash \mathscr{A}$ and hence $\mathscr{A}$ is not closed.

Suppose now, conversely, that (H3) is satisfied and define for $\rho>0$

$$
\mathscr{A}^{\rho}:=\left\{x\left|\left[b, t_{1}\right]\right| \exists u \in L_{p}:\|u\|_{p} \leqq \rho \text { and }(x, u) \text { satisfies }(4.1)\right\} .
$$

Let $\left(x^{k} \mid\left[b, t_{1}\right]\right)$ be a sequence in $\mathscr{A}^{\rho} \subset Z$ corresponding to a sequence $\left(u^{k}\right)$ in $\left\{u \in U:\|u\|_{p} \leqq \rho\right\}$ and converging to $z \in Z$. Since the spheres in $L_{p}$ are weakly compact, there is a subsequence $\left(u^{k_{i}}\right)$ of $\left(u^{k}\right)$ converging weakly to an element $u$ of $U$. By the continuity of the solution operator (3.14) $x\left(u^{k_{i}}\right)$ converges weakly to $x(u)$. Hence $z=x(u) \mid\left[b, t_{1}\right] \in \mathscr{A}^{\rho}$, because the limit is unique.

In the general case a sequence $\left(x^{k} \mid\left[b, t_{1}\right]\right)$ in $\mathscr{A}$ converging to $z \in W^{1, p}$ does not necessarily correspond to a bounded sequence of control functions. But, by appropriately generalizing Lemma 3.3 in [6] it is possible to derive, from (H2) and (H3), that there exists a bounded sequence of control functions yielding the same end functions $x^{k} \mid\left[b, t_{1}\right]$. Thus the general case may be reduced to the one just analyzed and the proposition is proved.

Proposition 4.3. $\mathscr{A}=W^{1, p}\left(\left[b, t_{1}\right], \mathbb{R}^{n}\right)$ iff $(\mathrm{H} 1),(\mathrm{H} 3),(\mathrm{H} 4)$ are valid.

Proof. Suppose (H1), (H3) and (H4) are satisfied. Then $\mathscr{A}$ is dense by Proposition 4.1 and closed by Proposition 4.2 since (H4) implies (H2).

Conversely, suppose $\mathscr{A}=W^{1, p}$. Then (H1) is satisfied. If (H4) is valid, (H3) follows by Proposition 4.2. It remains to prove the rank condition (H4). 
For each $z \in W^{1, p}$ there is $(x, u) \in X \times U$ satisfying

$$
\dot{z}(s)=\dot{x}(s)=A(s) x+B(s) u(s), \quad s \in\left[b, t_{1}\right] .
$$

We assume now that there is a compact subset $M \subset\left[b, t_{1}\right]$ of positive measure, such that Rank $B(s)<n$ for $s \in M$. Then there exists a measurable map $s \mapsto e(s)$ from $M$ into the unit sphere of $\mathbb{R}^{n}$ such that $B(s) B(s)^{*} e(s)=0$ and hence $B(s)^{*} e(s)=0$ a.e. on $M$ (cf. [32]). The map $A:\left[t_{0}, t_{1}\right] \rightarrow \underline{W}^{1, q}\left(\left[t_{0}, t_{1}\right], \mathscr{L}_{n n}\right)$ is measurable by assumption (3.10). Hence by Lusin's theorem (cf. [9]), there is a subset $N \subset M$ of positive measure such that $e \mid N$ and $A \mid N$ are continuous.

Let $\alpha$ be any function in $L_{p}(N, \mathbb{R})$. Define $f \in L_{p}\left(\left[b, t_{1}\right], \mathbb{R}^{n}\right)$ by

$$
f(s)= \begin{cases}0, & \text { for } s \in\left[b, t_{1}\right] \backslash N, \\ \alpha(s) e(s) & \text { for } s \in N\end{cases}
$$

and $z \in W^{1, p}\left(\left[b, t_{1}\right], \mathbb{R}^{n}\right)$ by

$$
z(s)=\int_{b}^{s} f(\tau) d \tau
$$

The scalar product of both sides of (4.6) with $e(s)$ yields, for a.e. $s \in N$,

$$
\langle\alpha(s) e(s), e(s)\rangle_{\mathbb{R}^{n}}=\langle A(s) x, e(s)\rangle_{\mathbb{R}^{n}}+\langle B(s) u(s), e(s)\rangle_{\mathbb{R}^{n}}
$$

i.e.,

$$
\begin{aligned}
\alpha(s) & =\langle A(s) x, e(s)\rangle_{\mathbb{R}^{n}}+\left\langle u(s), B(s)^{*} e(s)\right\rangle_{\mathbb{R}^{r}} \\
& =\langle A(s) x, e(s)\rangle_{\mathbb{R}^{n}} .
\end{aligned}
$$

This means that $\alpha$ is a.e. equal to a continuous function. This is a contradiction, because it may be shown that there is a function in $L_{p}(N, \mathbb{R})$ which is not almost everywhere equal to a continuous function.

COROllaRy. $\mathscr{A}$ is a dense proper subspace of $W^{1, p}\left(\left[b, t_{1}\right], \mathbb{R}^{n}\right)$, if $(\mathrm{H} 1),(\mathrm{H} 4)$ are satisfied and $(\mathrm{H} 3)$ is not.

Proposition 4.3 yields a full attainability criterion for HDS. In particular, we learn from it that it is impossible to steer hereditary differential systems from 0 to arbitrary target functions in $Z=W^{1, p}\left(\left[t_{1}-h, t_{1}\right], \mathbb{R}^{n}\right)$ if the number of input components $r$ is smaller than $n$, the number of state components. Since the condition $r \geqq n$ is rarely satisfied in practice, the concept of full attainability does not seem to be very practical. Evidently, the demand to hit exactly any target function in $W^{1, p}\left(\left[t_{1}-h, t_{1}\right], \mathbb{R}^{n}\right)$, by choosing appropriate control functions in $L_{p}\left(\left[t_{0}, t_{1}\right], \mathbb{R}^{r}\right)$, is too strong. Alternatively, the concept of approximate attainability (controllability) may be pursued (cf. e.g. [15], [29]). However, this concept means that $\mathscr{A}$ is dense in $Z$, and it is just this case which is difficult to handle by the maximum principle (see Theorem 1.1). Therefore Olbrot replaced the equality end constraint by the condition that the final state lies in a ball in a function space. Unfortunately it seems that his approach in [28] is not generalizable to systems with time-varying lag.

While Proposition 4.3 completely characterizes full attainability, there remains some distance between the necessary and the sufficient conditions for the closedness of $\mathscr{A}$. In particular, the rather strong assumption $(\mathrm{H} 2)$ is not necessary, as is shown by the following result of Kurcyusz-Olbrot [24]. 


\section{Proposition. Consider}

$$
\begin{gathered}
\dot{x}(t)=A_{1} x(t-1)+A_{2} x(t)+B u(t), \quad t \in\left[t_{0}, t_{1}\right], \\
x \mid\left[t_{0}-1, t_{0}\right]=0
\end{gathered}
$$

where $A_{1}, A_{2} \in \mathscr{L}_{n n}, B \in \mathscr{L}_{n r}, t_{1}-1>t_{0}, A_{1} \neq 0$, and $u \in L_{p}\left(\left[t_{0}, t_{1}\right], \mathbb{R}^{r}\right)$.

Then the attainable set $\mathscr{A}$ is closed in $W^{1, p}\left(\left[t_{1}-1, t_{1}\right], \mathbb{R}^{n}\right)$ iff $\operatorname{Im} A_{1} A_{2}^{i} B \subset \operatorname{Im} B$, $i=0,1, \cdots, n-1$.

Propositions 4.1-4.3 improve the results which have already been presented in the literature, expecially by Banks-Jacobs-Langenhop [6]. We generalize established results from HDS to Fredholm systems. Furthermore, Proposition 4.2 shows that (H3) is not only a sufficient [6, Thm. 3.3] but also a necessary condition for $\mathscr{A}$ to be closed (if $(\mathrm{H} 2)$ and (4.2) are assumed). This enables us to substitute $(\mathrm{H} 3)$ by the weaker assumption (4.2) as premise in the characterization of full attainability (compare Proposition 4.3 and [6, Thm. 3.1]).

\section{REFERENCES}

[1] H. T. BANKS, Variational problems involving functional differential equations, this Journal, 7 (1969), pp. 1-17.

[2] Control of functional differential equations with function space boundary conditions, Delay and Functional Differential Equations and their Application, K. Schmitt, ed., New York, 1972.

[3] H. T. BANKS AND M. Q. JACOBS, The optimization of trajectories of linear functional differential equations, this Journal, 8 (1970), pp. 461-488.

[4] - An attainable sets approach to optimal control of functional differential equations with function space boundary conditions, J. Differential Equations, 13 (1973), pp. 127-149.

[5] H. T. BANKS, M. Q. JACOBS AND C. E. LANGENHOP, Function space controllability for linear functional differential equations, Differential Games and Control Theory, E. O. Roxin, P. Liu, and R. L. Sternberg, eds., Marcel Dekker, New York-Basel, 1974, pp. 81-98.

[6] Characterization of the controlled states in $W_{2}^{(1)}$ of linear hereditary systems, this Journal, 13 (1975), pp. 611-649.

[7] H. T. BANKS AND G. A. KENT, Control of functional differential equations of retarded and neutral type with target sets in function space, this Journal, 10 (1972), pp. 567-593.

[8] H. T. BANKS AND A. MANitius, Application of abstract variational theory to hereditary systems-A survey, IEEE Trans. Automatic Control, AC-19 (1974), pp. 524-533.

[9] H. BAUER, Wahrscheinlichkeitstheorie und Grundzüge der Maßtheorie, 2. Auflage, De Gruyter, Berlin, 1974.

[10] Z. BIEN, Optimal control of delay systems, Ph.D. thesis, University of Iowa, Ames, 1975.

[11] D. H. CHYung AND E. B. LEE, Delayed action control systems, Automatica, 6 (1970), pp. 395-400.

[12] F. COlOniUs AND D. HINRICHSEN, Optimal control of hereditary differential systems, Part I, Arbeitspapiere Mathematik Nr. 2, Univ. Bremen, Bremen, W. Germany, 1976.

[13] R. Courant AND D. Hilbert, Methoden der Mathematischen Physik, Springer-Verlag, Berlin, 1968.

[14] M. C. Delfour AND A. MANitius, Control systems with delays: Areas of applications and present status of the linear theory, Rep. CRM-658, Univ. Montréal, 1976.

[15] M. C. Delfour AND S. K. MITTER, Controllability, observability and optimal feedback control of affine hereditary differential systems, this Journal, 10 (1972), pp. 298-328.

[16] R. D. DRIVER, A "backwards" two-body problem of classical relativistic electrodynamics, Phys. Rev., 178 (1969), pp. 2051-2057.

[17] N. Dunford AND J. T. SCHWARTZ, Linear Operators, Part I: General Theory, Wiley-Interscience, New York, 1967.

[18] I. V. GiRsANOV, Lectures on Mathematical Theory of Extremum Problems, Springer-Verlag, Berlin, 1972.

[19] J. HAlE, Theory of Functional Differential Equations, 2nd ed., Springer-Verlag, New York, 1977.

[20] H. HALKIN, A satisfactory treatment of equality and inequality constraints in the Dubovitskii-Milyutin optimization formalism, J. Optimization Theory Appl., 6 (1970), pp. 138-149. 
[21] M. Q. JACOBS AND T. J. KAO, An optimum settling problem for time-lag systems, J. Math. Anal. Appl., 40 (1972), pp. 687-707.

[22] S. KURCYUSZ, A local maximum principle for operator constraints and its application to systems with time lag, Control and Cybernetics, 2 (1973), pp. 99-125.

[23] — On the existence and nonexistence of Lagrange multipliers in Banach spaces, J. Optimization Theory Appl., 20 (1976), pp. 81-110.

[24] S. KURCYUSZ AND A. W. OLBROT, On the closure in $W^{1, a}$ of the attainable subspace of linear time lag systems, J. Differential Equations, 24 (1977), pp. 29-50.

[25] D. G. Luenberger, Optimization by Vector Space Methods, John Wiley, New York, 1968.

[26] K. MAKOWSKI AND L. W. NEUSTADT, Optimal control problems with mixed control phase variable equality and inequality constraints, this Journal, 12 (1974), pp. 184-228.

[27] L. W. Neustadt, Optimization-A Theory of Necessary Conditions, Princeton University Press, Princeton, NJ, 1976.

[28] A. W. Olbrot, Control of retarded systems with function space constraints; Necessary optimality conditions, Control and Cybernetics, 5 (1976), pp. 5-31.

[29] - Control of retarded systems with function space constraints, Part 2: Approximate controllability, Ibid., 6 (1977), pp. 17-71.

[30] R. Penrose, A generalized inverse for matrices, Proc. Cambridge Philos. Soc., 51 (1955), pp. 406-413.

[31] L. S. Pontryagin, V. G. Boltyanskil, R. V. Gamkrelidze and E. F. Mischenko, The Mathematical Theory of Optimal Processes, John Wiley, New York, 1962.

[32] W. T. REID, Some elementary properties of proper values and proper vectors of matrixfunctions, SIAM J. Appl. Math., 18 (1970), pp. 259-266.

[33] M. M. VAInberg, Some problems in the differential calculus in linear spaces, Uspehi Mat. Nauk, 7 (1952), No. 4, pp. 55-102. (In Russian.)

[34] - Variational Methods for the Study of Nonlinear Operators, Holden-Day, San Francisco, 1964.

[35] H. VAN DAM AND E. P. WIGNER, Classical relativistic mechanics of interacting point particles, Phys. Rev. B, 138 (1965), pp. 1576-1582.

[36] J. Warga, Optimal Control of Differential and Functional Equations, Academic Press, New YorkLondon, 1972.

[37] - Optimal controls with pseudo-delays, this Journal, 12 (1974), pp. 286-299.

[38] L. V. WOLFERSDORF, Optimale Steurungsprobleme bei linearen Integralgleichungen, I. Fredholmsche Integralgleichungen, Beiträge Anal., 7 (1975), pp. 113-130.

[39] A. C. ZAAnEN, Linear Analysis, North-Holland, Amsterdam, 1964.

[40] P. P. ZABREYKO ET AL., Integral Equations-A Reference Text, Noordhoff, Leyden, 1975. 
Reproduced with permission of the copyright owner. Further reproduction prohibited without permission. 